\title{
Cochlear Damage Caused by the Striking Noise of Titanium Head Golf Driver
}

\author{
Yong-Ho Park ${ }^{1, *} \cdot$ Juyong Chung ${ }^{2, *} \cdot$ Min Young Lee ${ }^{3}$ Doh Young Lee $\cdot$ Young Ho Kim \\ ${ }^{1}$ Department of Otolaryngology-Head and Neck Surgery, Brain Research Institute, College of Medicine, Chungnam National University, Daejeon; \\ ${ }^{2}$ Department of Otolaryngology-Head and Neck Surgery, Wonkwang University College of Medicine, Iksan; \\ ${ }^{3}$ Department of Otorhinolaryngology-Head and Neck Surgery, Dankook University College of Medicine, Cheonan; \\ ${ }^{4}$ Department of Otorhinolaryngology-Head and Neck Surgery, SMG-SNU Boramae Medical Center, Seoul, Korea
}

Objectives. To investigate how mouse cochleae are affected by the striking noise of titanium head golf driver.

Methods. Thirty-two BALB/c mice (20-22 g) with normal hearing were used. The impact acoustic stimulus generated by the striking of titanium golf driver head was centered around $4.5 \mathrm{kHz}$ with $120.5 \mathrm{~dB}$ sound pressure level. The recorded impact noise was provided to mice in two ways with the same exposure time of 288 seconds: 1,440 repetitions and an impact duration of 0.2 seconds for 2 hours (repetitive noise) or serially connected impact noise for 288 seconds (continuous noise). Auditory brainstem responses were measured at baseline, day 7, and day 14 after exposure. The mice were then sacrificed for histology.

Results. Both groups showed statistically significant threshold shifts immediately after noise exposure. Mice in the continuous exposure group, except for those exposed to $32 \mathrm{kHz}$ noise, recovered from threshold shifts 1-2 weeks after noise exposure. However, in the repetitive exposure group, threshold shifts remained for 2 weeks after exposure. The repetitive exposure group had greater hair cell damage than did the continuous exposure group. Structural changes in the stria vascularis were observed in the repetitive exposure group.

Conclusion. Overexposure to impact noise caused by hitting of titanium head golf driver may be hazardous to the cochlea, and repetitive exposure may induce greater damage than continuous exposure.

Keywords. Hearing Loss; Noise-Induced; Titanium; Golf; Cochlea

\section{INTRODUCTION}

To improve their striking distances, most golfers practice swinging their golf clubs for long durations. Recently, many people enjoy games or practices in indoor golf practice booths where the noise generated by striking a ball is more condensed than in outdoor practice ranges. However, most of people using indoor

- Received November 29, 2017

Revised January 31, 2018

Accepted February 22, 2018

- Corresponding author: Young Ho Kim

Department of Otorhinolaryngology-Head and Neck Surgery, SMG-SNU

Boramae Medical Center, 20 Boramae-ro 5-gil, Dongjak-gu, Seoul 07061, Korea

Tel: +82-2-870-2442, Fax: +82-2-870-3863, E-mail: yhkiment@gmail.com

*The first two authors contributed equally to this study. practice booths are not aware of risk of noise exposure although there have been several studies showing that the impact sound caused by striking a ball with a golf club has been implicated in hearing damage $[1,2]$. We recently analyzed the characteristics and the auditory hazards of impact noise caused by titanium golf driver heads (GDHs), and demonstrated that impact noise generated by the striking of titanium head golf drivers against balls showed a high peak level of approximately $120 \mathrm{~A}$-weighted $\mathrm{dB}(\mathrm{dBA})$ and a very short duration of less than $200 \mathrm{~ms}$ which could be hazardous to those frequently exercise gold in indoor booth [3].

Overstimulation by sound commonly causes cochlear damage leading to sensorineural hearing loss; this damage is called noiseinduced hearing loss (NIHL). Noise that can induce hearing loss is divided into two categories: continuous and impulse [4]. Many

Copyright @ 2019 by Korean Society of Otorhinolaryngology-Head and Neck Surgery.

This is an open-access article distributed under the terms of the Creative Commons Attribution Non-Commercial License (http://creativecommons.org/licenses/by-nc/4.0)

which permits unrestricted non-commercial use, distribution, and reproduction in any medium, provided the original work is properly cited. 
studies have proved the mechanism how continuous noise affects the cochlea [4-10]. The main mechanism is thought to comprise oxidative stress after noise exposure including elevated levels of reactive oxygen species, reactive nitrogen species, and free radicals [9]. However, cochlear damage after exposure to impact or impulse noise has not been well studied, and the mechanism by which this damage occurs is not fully understood [4]. The aim of this study was to investigate cochlear damage by the noise generated from striking of titanium head golf driver using the evoked auditory potentials and cochlear histological analysis.

\section{MATERIALS AND METHODS}

\section{Animals and noise exposure}

Thirty-two BALB/c mice (20-22 g) with normal hearing were used in this study. The sound generated by a titanium GDH striking a ball in real environment was used as the source sound, as described in a previous report [3]. Two types of noises, repetitive and continuous, were produced from the source sound. Assuming that we usually practice golf in indoor range for 2 hours $(1,440$ repetitions), the 16 mice were exposed to a repetitive impact sound that was centered at $4.5 \mathrm{kHz}$ and had a peak sound pressure level (SPL) of $120 \mathrm{~dB}$, a repetition rate of once per 5 seconds and a duration of 0.2 seconds (Fig. 1). For the same acoustic energy during the 2 hours of indoor golf practice, the other 16 mice were exposed to the continuous version of the source sound with the same speculation for 288 seconds. The repetitive impact sound (repetition rate, once per 5 seconds; duration, 0.2 seconds) was generated for 2 hours $(7,200$ seconds), and total exposure time of noise was 288 seconds $(7,200 \mathrm{sec} / 5 \mathrm{sec} \times 0.2$ $\mathrm{sec})$. All experiments were performed in an acoustically insulated booth. The noise signals were routed through a computer and an amplifier (INTER-M R300 Plus Power Amplifier; Canford Audio, Washington, UK) to a loudspeaker (ElectoVoice DH1AWP; Sonic Electronix, Sylmar, CA, USA). The noise level was measured at mouse cage with a sound level meter (Type 2250;

\section{H I G G H L I G G H T S}

- Mice with continuous exposure of striking noise recovered from threshold shifts 1-2 weeks after noise exposure.

- Mice with repetitive exposure of striking demonstrated noise threshold shifts remained for 2 weeks after exposure.

- The repetitive exposure resulted in greater hair cell damage than the continuous exposure.

- Overexposure to impact noise caused by hitting of titanium head golf driver may be hazardous to the cochlea, and repetitive exposure may induce greater damage than continuous exposure.
Brüel \& Kjaer, Naerum, Denmark), a sound calibrator (Type 4231, Brüel \& Kjaer), and a condenser microphone (Type 4189, Brüel \& Kjaer). All procedures, including those for the use and care of the animals, were approved by the animal experiment committee.

\section{Auditory brainstem response measurement}

Auditory brainstem responses (ABRs) used to assess hearing thresholds, were measured before noise exposure, immediately after noise exposure, day 7, and day 14 after noise exposure. The changes in ABR thresholds before and after noise exposure were compared.TDT System-3 (Tucker-Davis Technologies, Gainesville, FL, USA) hardware and software were used to obtain ABRs with 1,000 stimulus repetitions per record. Mice were anesthetized with a mixture of ketamine $(40 \mathrm{mg} / \mathrm{kg})$ and xylazine $(10 \mathrm{mg} / \mathrm{kg})$ and kept warm with a heating pad during ABR recordings. Platinum needle electrodes were placed subcutaneously on the vertex (positive), ipsilateral (negative) ear, and contralateral (ground) ear. Through an inset speculum in the external auditory meatus, click sounds and tone bursts with durations of $4 \mathrm{~ms}$ and rise-fall times of $1 \mathrm{~ms}$ at frequencies of $8 \mathrm{kHz}, 16$ $\mathrm{kHz}$, and $32 \mathrm{kHz}$ were then presented to the right ear. The sound intensity was varied by $10 \mathrm{~dB}$ intervals for the tone-burst sounds and by $5 \mathrm{~dB}$ intervals for click sounds near the threshold.

\section{Immunocytochemistry with surface preparation of organ of Corti}

Mice were sacrificed on day 14 after noise exposure, and the cochleae were harvested. The stria vascularis, the spiral ligament, and tectorial membrane were dissected from the organ of Corti. Then, the organ of Corti was separated into three regions: the apical, middle, and basal turns. The organ of Corti samples were fixed in $2 \%$ paraformaldehyde in phosphate-buffered saline (PBS) for 15 minutes at room temperature. After incubation in $0.25 \%$ Triton X-100 for 2 minutes, the specimens were rinsed with PBS and immersed in tetramethylrhodamine isothiocyanate-labeled phalloidin (1:100 diluted; Sigma, St. Louis, MO, USA) in PBS for 20 minutes. Cells were then fixed in 3.7\% paraformaldehyde for 10 minutes at room temperature. After being washed with PBS, cells were observed under a fluorescent microscope (IX71; Olympus, Tokyo, Japan).

\section{Scanning electron microscope examination}

Intracardiac perfusions were performed, and the cochleae were removed immediately. The cochleae were then decalcified in rapid decalcification solution for 20 minutes, after which the bony capsule was removed and the lateral wall tissues (spiral ligament and stria vascularis) and the membranous structure were separated under a dissecting microscope. The dissected specimens were rinsed with $0.1 \mathrm{M}$ Dulbecco's PBS (DPBS) and postfixed in $1 \% \mathrm{OsO}_{4}$ for 15 minutes. The specimens were gently rinsed with 0.1 M DPBS again and dehydrated in graded ethanol solu- 


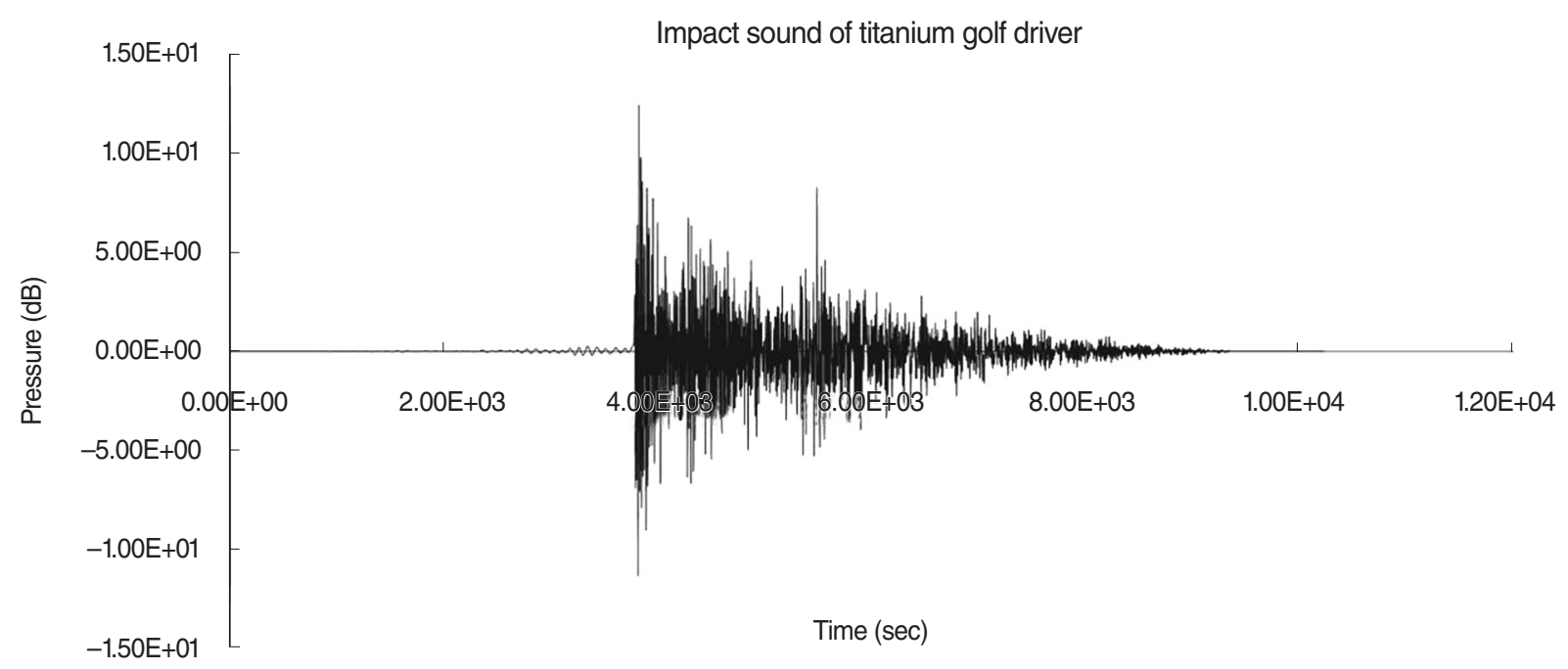

Fig. 1. Acoustic spectrum of variation of titanium golf driver head impact noise. This figure is an example of the sound stimulus applied in this study. The repetitive impact noise, which lasted for 2 hours (1,440 repetitions), is centered at $4.5 \mathrm{kHz}$ and has a peak sound pressure level of about $120 \mathrm{~dB}$, a repetition rate of once per 5 seconds, and a duration of 0.2 seconds.

tions. They were placed in hexamethyldisilazane for 15 minutes and dried at room temperature overnight. The dried specimens of the organ of Corti were attached to aluminum stubs with aluminum paint and then coated with platinum-palladium by using E-1030 PT-PD target assembly (Hitachi, Tokyo, Japan). The surfaces of the organs of Corti were examined with an S-4300 scanning electron microscope (SEM; Hitachi, Tokyo, Japan).

\section{Transmission electron microscope examination}

The cochleae were postfixed in $1 \% \mathrm{OsO}_{4}$ after being immersed in a $2.5 \%$ glutaraldehyde solution. They were then decalcified, osmicated, and dehydrated in an ethanol series. Next, the cochleae were embedded in epoxy resin. After polymerization, samples were sectioned on a Leica EMU6 ultramicrotome. Sections were stained with toluidine blue in accordance with standard transmission electron microscope (TEM) procedures, and TEM images were acquired (Tecnai G2 Spirit Twin; FEI Company, Hillsboro, OR, USA).

\section{Statistical analysis}

The changes in ABR thresholds before and after noise exposure were analyzed by using the paired $t$-test. The $P$-values $<0.05$ were considered statistically significant. Statistical analysis was conducted with SPSS ver. 17.0 (SPSS Inc., Chicago, IL, USA).

\section{RESULTS}

\section{ABR threshold shifts after noise exposure}

Both groups showed statistically significant threshold shifts after noise exposure $(P<0.05)$. In the continuous group, there were statistically significant threshold shifts for the click stimulus,
$8-\mathrm{kHz}, 16-\mathrm{kHz}$, and $32-\mathrm{kHz}$ frequency stimuli $(P<0.05)$. One week after continuous noise exposure, complete recoveries were seen at the clicks and $8 \mathrm{kHz}$ and $16 \mathrm{kHz}$, but recoveries at 32 $\mathrm{kHz}$ were partial. Significant shifts in hearing thresholds remained for 2 weeks after exposure at $32 \mathrm{kHz}(P<0.05)$ (Fig. 2A). In the repetitive group, threshold shifts were seen for all stimulus levels, and these shifts remained for 2 weeks. Thresholds at 1 week were statistically lower than the thresholds just after noise exposure at $16 \mathrm{kHz}, 32 \mathrm{kHz}$, and click sound; recoveries at all stimulus levels were partial $(P<0.05)$. Thresholds at 2 weeks were statistically lower than those just after exposure at $8 \mathrm{kHz}, 16 \mathrm{kHz}$, click sound $(P<0.05)$. ABRs 2 weeks after noise exposure revealed statistically significant threshold shifts at all stimulus levels $(P<0.05)$ (Fig. 2B). The mean threshold of the repetitive group was $11.5 \mathrm{~dB}$ higher than that of the continuous group (58.1 dB vs. $46.6 \mathrm{~dB}$ ). The absolute value of the threshold shift of repetitive group was larger than that of the continuous group (33.2 $\mathrm{dB}$ vs. $20.6 \mathrm{~dB}$ ). Average ABR thresholds at the baseline, immediately after noise exposure, 1 week later, and 2 weeks later are shown in Fig. 2.

\section{Immunocytochemistry findings}

The epifluorescent immunocytochemistry findings were consistent with the $\mathrm{ABR}$ results. In the continuous group, the rhodamine-phalloidin staining of the organ of Corti showed an orderly outline of three rows of outer hair cells and one row of inner hair cells in the cochlear apical turn (Fig. 3A). Some outer hair cell loss was found in the cochlear middle and basal turns (Fig. $3 \mathrm{~B}, \mathrm{C})$. The repetitive group showed greater outer hair cell loss in all turns of the cochlea and definite deterioration near the basal turn (Fig. 3D-F). 

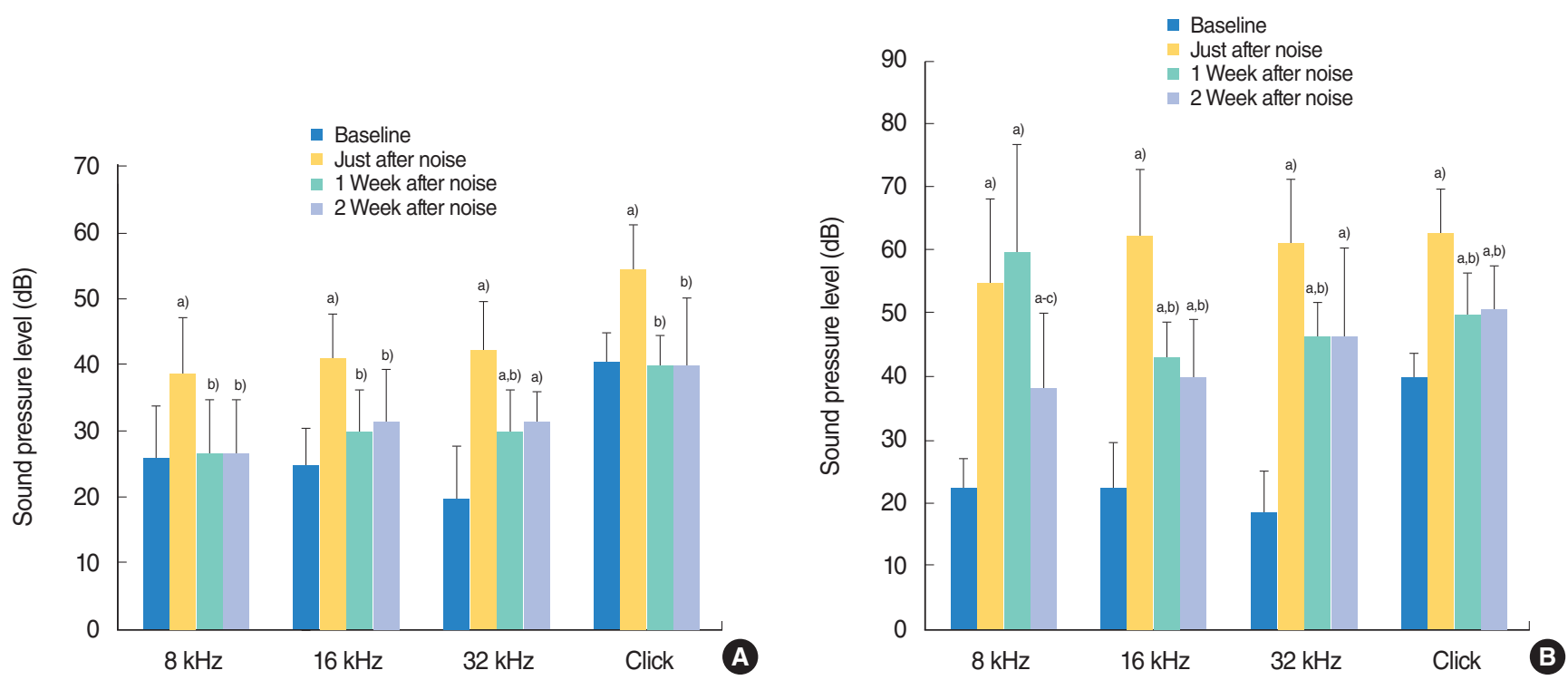

Fig. 2. Hearing threshold changes after exposure to continuous noise $(A)$ and repetitive impact noise (B). In the continuous exposure group, statistically significant threshold shifts were found immediately after noise exposure at the click, 8-kHz, 16-kHz, and 32-kHz tonal stimulus. One week after exposure, recoveries from threshold shifts at the clicks, $8 \mathrm{kHz}$ and $16 \mathrm{kHz}$ were complete. Permanent threshold shifts were confirmed 2 weeks after exposure at $32 \mathrm{kHz}(\mathrm{A})$. In the repetitive exposure group, statistically significant threshold shifts were found immediately after exposure at the clicks and each frequency. Permanent threshold shifts were confirmed 2 weeks after exposure (B). ${ }^{\text {a) } T h e ~ d i f f e r e n c e ~ f r o m ~}$

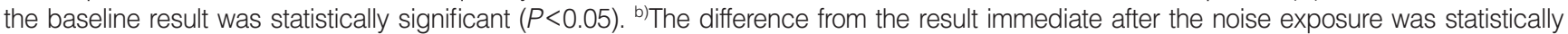
significant $(P<0.05)$. ${ }^{\mathrm{c}}$ The difference from the result 1 week after noise exposure was statistically significant $(P<0.05)$.
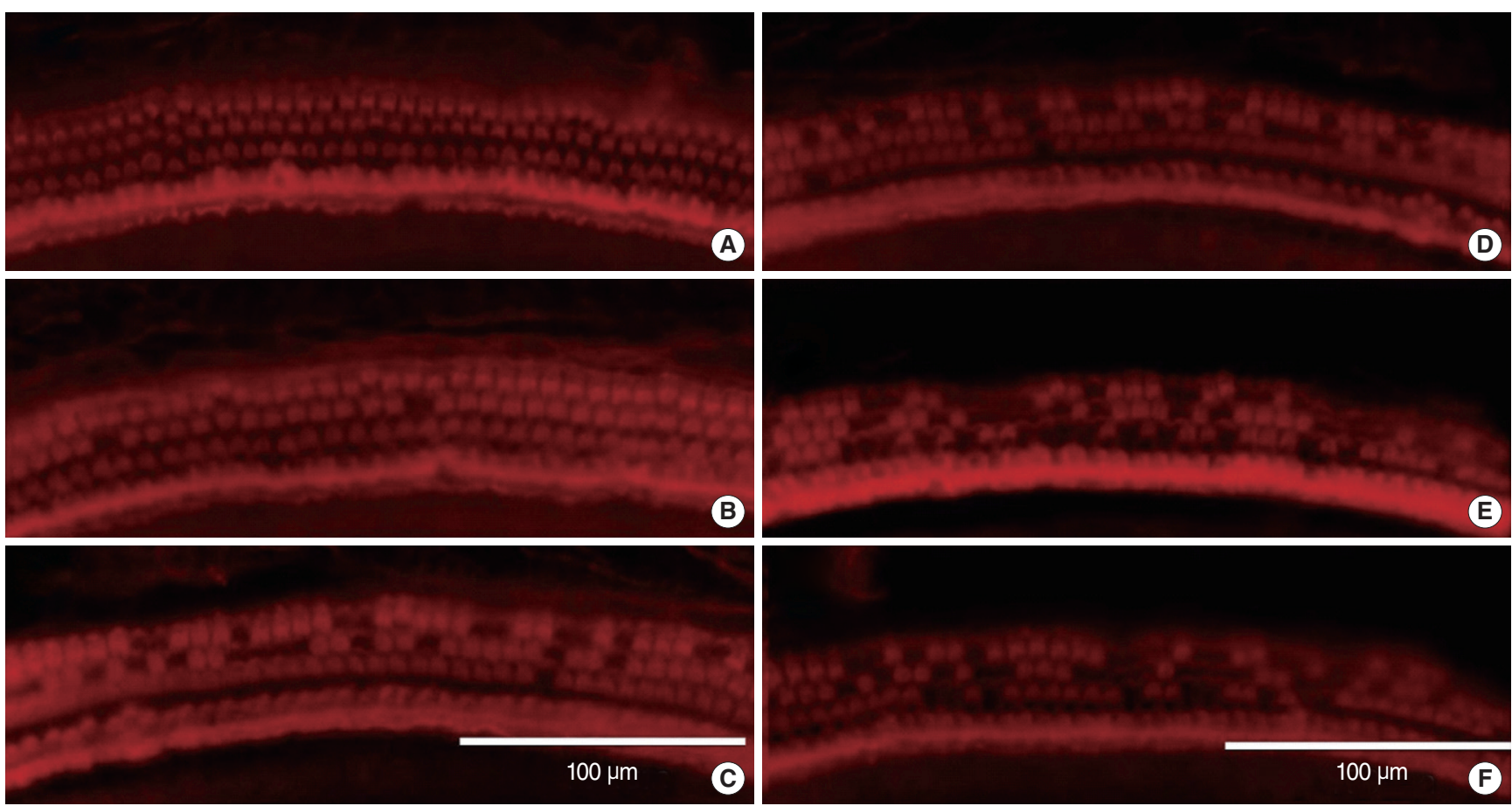

Fig. 3. Epifluorescent immunocytochemistry analysis of organs of Corti: continuous noise exposure group (A-C) and repetitive noise exposure group (D-F). In the continuous impact noise exposure group, the three rows of outer hair cells and one row of inner hair cells were orderly and preserved in the cochlear apical turn (A). Some outer hair cell loss was observed in the middle and basal turns (B, C). In the repetitive impact noise exposure group, the disappearance of the orderly structural arrangement in the organ of Corti was more apparent in all turns of the cochlea, and deterioration was observed near the basal turn. More hair cells were preserved in the apical turn than in the basal one (D); outer hair cell loss was more severe in the basal turn (F). 

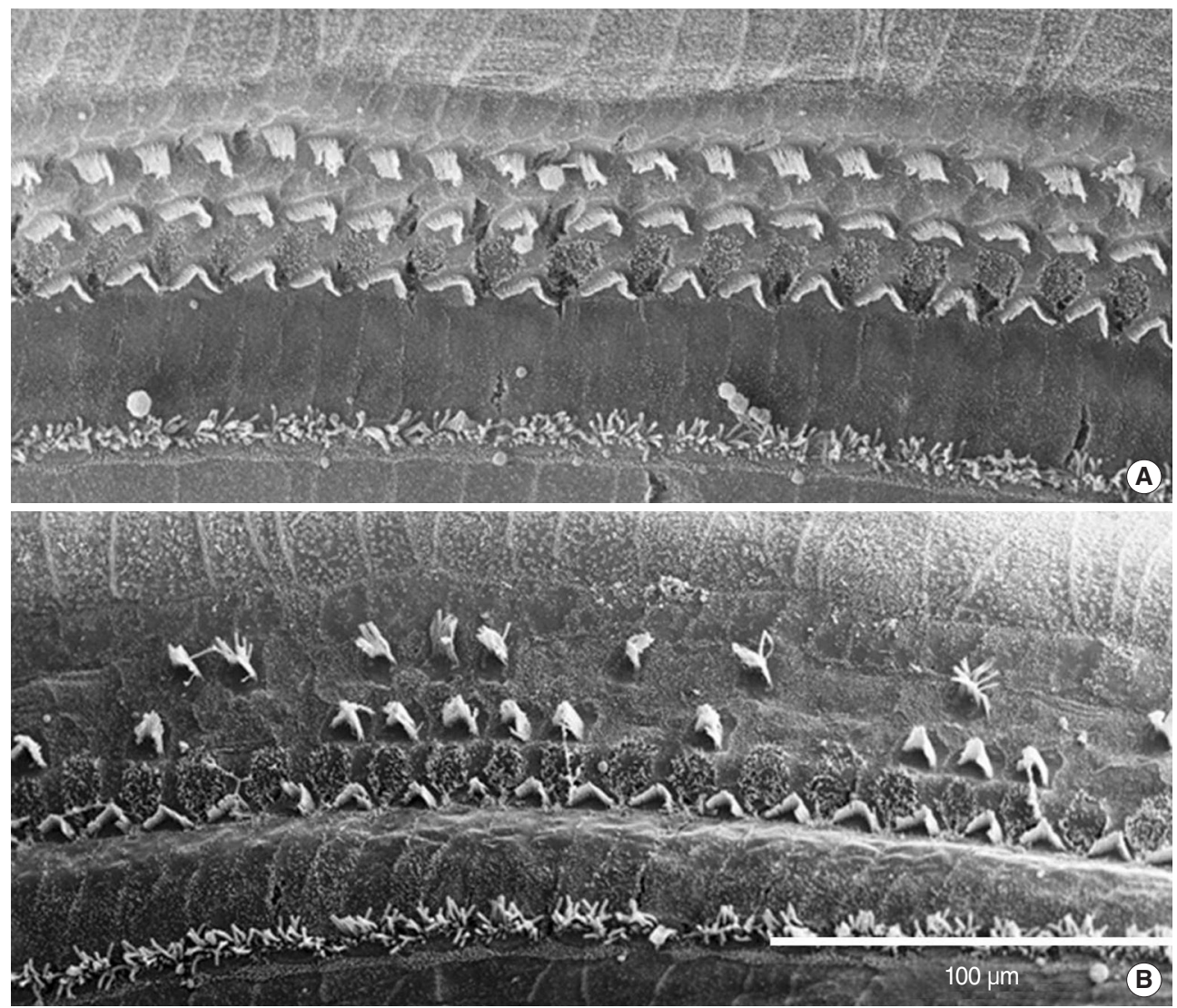

Fig. 4. Scanning electron microscope images of organs of Corti: continuous noise exposure group (A) and repetitive noise exposure group (B). In the continuous noise exposure group, the structures of outer hair cells in the apical turn of the organ of Corti were normal (A). However, in the repetitive noise exposure group, the loss of outer hair cells and disorganization of stereocilia among the remnant outer hair cells were observed in the apical turn of the organ of Corti (B).

\section{SEM findings}

The findings from the apical turns were similar to the epifluorescent immunocytochemistry results. In the continuous group, the hair cells in the organ of Corti were morphologically normal and undamaged (Fig. 4A). In the repetitive group, the outer hair cell damage extended to the apical turn. The outer two rows of the outer hair cells were damaged; however, the innermost row of outer hair cells was spared. In addition, irregularly organized stereocilia were visible in the remaining outer hair cells (Fig. 4B).

\section{TEM findings}

In the repetitive group, shrunken and vacuolized intermediate cells and large intercellular spaces were observed in the stria vascularis. However, in the continuous group, the stria vascularis appeared nearly normal (Fig. 5).

\section{DISCUSSION}

In this study, the impact sound generated when one strikes a ball with a titanium GDH was used as a source of NIHL. This is not a common stimulus as noise source in NIHL research. Therefore, defining and categorizing the stimulus sound used in the present study seems necessary. As described in the introduction, noise can be classified into continuous noise and intermittent (impulse) noise, and impulse noise can be defined as short duration sound with a nearly instantaneous rise time [11]. Impulse noise is classified into two categories: subsonic (impact noise) and supersonic (blast noise) [12]. Blast noises are created by explosive devices and artillery with overpressures that are faster than sound speed. Impact noise is defined as a repetitive sound burst that is louder than ambient sound by $15 \mathrm{~dB}$ and occurs at 1 second intervals [13]. According to these classifications, the golf driver's striking sound can be defined as an impact sound. In past decades, a number of studies regarding NIHL after blast noise were performed to examine symptoms of war veterans or former industrial employees whose work environments contained excessive blast noises [14-22]. However, studies regarding hearing loss due to impact noise are less common in the field of otolaryngology, and fewer articles regarding leisure activities were reported.

One of the main findings in this study is the confirmation of hearing loss and cochlear damage after exposure to the noise 

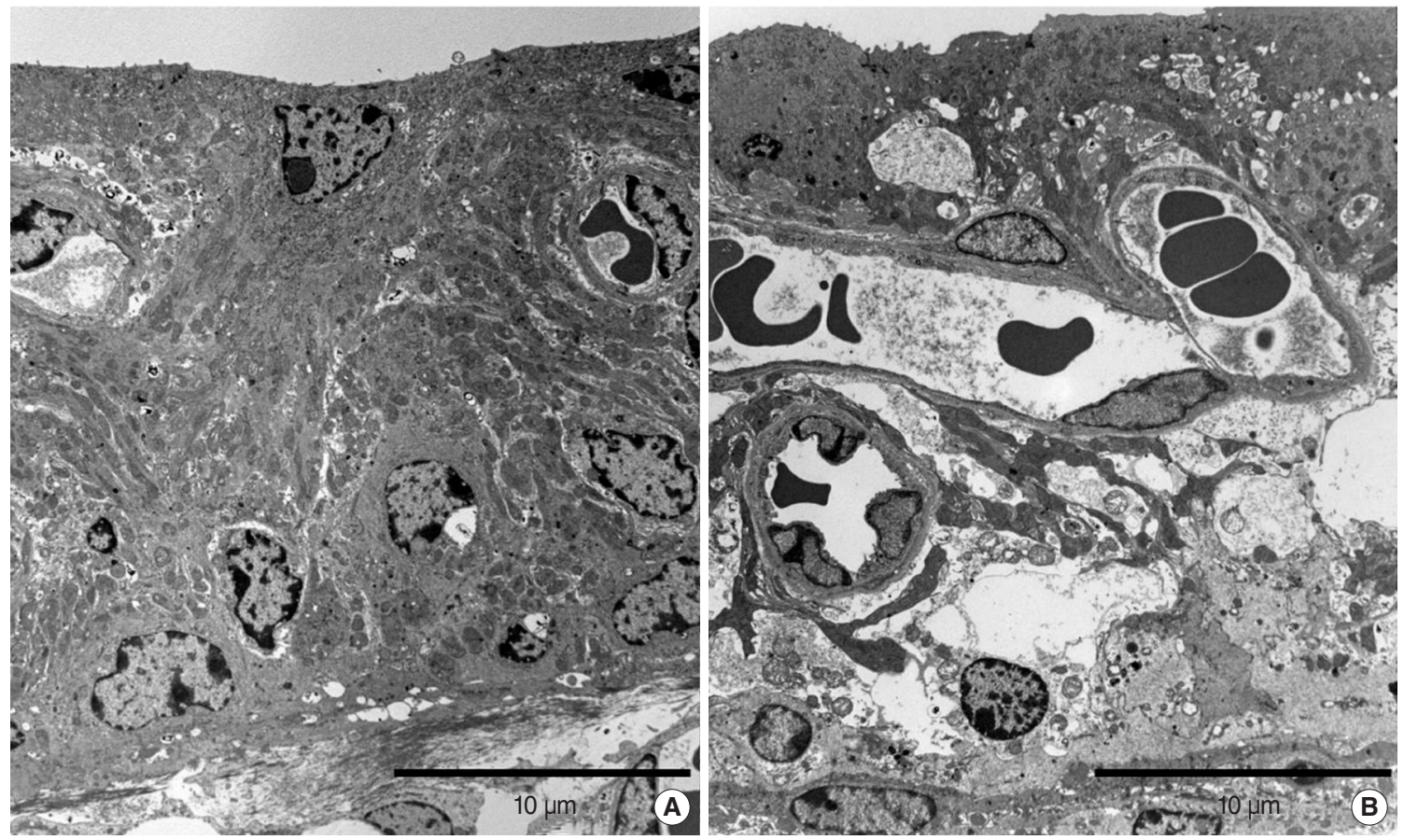

Fig. 5. Transmission electron microscope images of the apical turn of the stria vascularis of the continuous noise exposure group (A) and that of the repetitive noise exposure group (B). Normal findings were observed in the continuous noise exposure group (A). Shrunken and vacuolized intermediate cells and large intercellular spaces were visible in repetitive noise exposure group (B).

generated from hitting by a titanium GDH. In this study, cochlear hair cell damages as well as changes in evoked potential thresholds were identified. Previous reports suggested the possibility of hearing loss or presented cases of hearing loss caused by the impact sound of a golf driver. Buchanan et al. [1] have reported one case of NIHL presenting after golf was played as a leisure activity. As another study, we analyzed the acoustic characteristics of the noise caused by titanium GDHs from various companies and suggested that the impact noise might create potential auditory hazards [3]. More recently, with the real ear measurement system, Zhao and Bardsley [2] analyzed the effect of the golf driver impact sound and suggested that hearing damage could result from this noise. They showed that golf driver's noise greater than $116 \mathrm{dBA}$ poses a significant risk of NIHL [2]. However, to the best of our knowledge, the present study is the first report confirming, both functionally and histologically, that the impact sound caused by a titanium GDH could cause noise-induced cochlear damage. Without hearing protection, the longterm exposure to this type of impact noise may cause permanent NIHL. This result may raise alarm among people who spend time in intensely noisy environments such as golf courses or loud music venues. To prevent NIHL, the impact noises encountered in daily life should be examined, and the public should be educated about programs to avoid noise exposure. Hearing protection devices and future medicine for preventing and reducing hearing loss after noise exposure are possible solutions to prevent NIHL.
Another finding of the present study is that repetitive exposure caused more functional and histopathologic damages than continuous exposure. Although the length of exposure time was similar between the two groups, the functional and histopathologic outcomes differed. Impact noise has been suggested to induce less hearing loss than continuous noise at a noise emission level <115 dBA [3]. However, it becomes more hazardous to hearing at emission levels $>115 \mathrm{dBA}$ [3]. In our study, the impact sound had a peak SPL of $120 \mathrm{~dB}$ and was centered at 4.5 $\mathrm{kHz}$, and repetitive exposure caused more cochlear damage than continuous exposure.

In continuous noise exposure, recoveries were complete at all frequencies at 1 week after noise exposure. However, in repetitive noise exposure, threshold shifts were remained at all frequencies for 2 weeks after noise exposure and recoveries were partial. Even though low frequency $(8 \mathrm{kHz})$ was more deleterious at 1 week after repetitive noise exposure, the threshold at 2 weeks were statistically lower than that at 1 week. However, high frequencies showed constant threshold shifts after 1 week. In conclusion, larger threshold shifts were shown at high frequencies. In addition, our result is consistent with that of a previous animal study in which chinchillas were exposed to impulsive noise and continuous noise [12]. Chinchillas exposed to continuous noise sustained a temporary threshold shift, then recovered linearly after noise cessation [12]. However, exposure to impulse noise with the same total energy led to greater thresholds shifts for 3 hours after noise cessation. Moreover, compared to contin- 
uous noise, repetitive noise resulted in more prolonged and less complete recoveries. In addition, human studies corroborated these results [12]. Data from industries and the military demonstrate that cochlear damage due to impulse noise is more extensive than the damage caused by continuous noise with same total energy level [12]. Furthermore, previous studies suggested that the degree of hearing loss after impact noise exposure does not seem to be correlated with peak SPL, exposure time length [23-25], exposure level dosimetry [26,27], equivalent energy level, blast numbers, or different spectra of energy [28]. There were trials to redefine outcomes of this stimulus type in the past. The National Institute for Occupational Safety and Health has decided that the equal energy hypothesis is not applicable to impulse NIHL $[12,23]$. The reason for this is still poorly understood. Impact noise is different from continuous noise, and it seems difficult to correlate hearing loss with the amount of impact noise [12].

The mechanisms for inner ear damage may differ according to the type of noise. It had been proposed that impulse and continuous noises affect the inner ear differently [29]. Inner and outer hair cells were affected differently by impulse noise because the two types of noises initiate different modes of movement along the basilar membrane, and outer hair cells would be affected more [29]. This suggestion is consistent with the results of the present study, in which outer hair cells were damaged more severely, as shown by epifluorescence immunocytochemistry and SEM imaging analysis (Figs. 3, 4). However, this could also be explained by the difference in mechanisms induced by the two stimuli. During continuous noise exposure, reactive oxygen species and reactive nitrogen species are produced by the motor metabolism of outer hair cells, and these are cleared by glutathione peroxidase and other natural scavengers [30-33]. Excessive noise elevates the concentration of reactive metabolites; if the natural scavenger concentration is insufficient to clear the metabolites, these free radicals can damage DNA, proteins, lipids, and cell membranes [34-36]. However, in the case of impact NIHL, mechanisms other than these biochemical reactions could be occurring. When the stimulus level is low, the same adverse biochemical reactions take place to damage cochlear hair cells, whereas noise $>125 \mathrm{~dB}$ might lead to the passive movement of the basilar membrane and the mechanical damage of the cochlea [23]. The mechanical component of the damaging process seems to start with the loss of stereocilia and the avulsion of the basilar membrane and extend to the damage of outer hair cells [37, 38]. Impulse noise with the intensity of $155 \mathrm{~dB}$ created direct and immediate mechanical damage in chinchilla cochlea [39]. And, the cochlear pathology can induce a disruption of the mechanical support of the pillar cells, ripping apart the tight cell junctions, and in the extreme, the separation of the organ of Corti from basilar membrane [39]. In other study, guinea pig cochleae following exposure to $167 \mathrm{~dB}$ impulses showed noise-induced outer hair cell loss [40]. Some histopathologic findings in the present study support their suggestions. SEM images show that remnant outer hair cells that survived repetitive noise exposure had disorganized stereocilia (Fig. 4).

Additionally, TEM images of the stria vascularis reveal shrunken cells and large intercellular spaces (Fig. 5). The atrophy of stria vastularis caused by ototoxic drug-induced cochlear damage was reported in some studies [41-43]. In addition, the generation of reactive oxygen species is thought to be part of the mechanism underlying NIHL. Superoxide anion radicals emerge in the stria vascularis after intense noise exposure [44]. These damages to the outer hair cells suggested the possibility that mechanical injury results from passive basilar membrane movement. The stereocilia on the outer hair cells are displaced in proportion to the intensity of the energy propagation inside the scala media [12]. The passive component of basilar membrane vibration might injure the cochlea through purely mechanical damage. The histologic change of the cochlea after impact noise exposure has been demonstrated [12]. After exposure to $119 \mathrm{~dB}$ impulse noise for 30 hours, outer hair cells demonstrated immediate splaying and loss of stereocilia. After exposure to $131 \mathrm{~dB}$ impulse noise for 1.8 hours, additional damage to outer hair cells was observed: holes in the reticular lamina and ruptures of cell-to-cell contacts of pillar cells were seen in a wider area of the cochlea than in the area affected by the initial impact frequency. The researchers reported that the organ of Corti tore at $137 \mathrm{~dB}$ and that strips of sensory epithelium were avulsed from the basilar membrane at $160 \mathrm{~dB}$.

In the present study, there were some limitations. First, noiseinduced hearing impairment in mice did not recover to the preexposure hearing levels in either the repetitive group or the continuous group. Therefore, further comparative studies regarding hearing recovery in the two groups are needed. Second, mice cochleae do not reflect all of the acoustic characteristics of human cochleae. Research on humans will be necessary to investigate human cochlear damage caused by impact noise. Third, the impact noises used in this study are not same as those encountered in daily living. To prevent hazardous noises that occur for various reasons, including occupational and leisure activities, evidence-based noise studies should be performed in the future.

The impact sound generated by a titanium GDH striking a ball, whether repetitive or continuous, may lead to cochlear damage. In addition, when animals are exposed to similar amount of noise duration, repetitive noise may induce more harmful cochlear damage than nonrepetitive noise.

\section{CONFLICT OF INTEREST}

No potential conflict of interest relevant to this article was reported. 


\section{ACKNOWLEDGMENTS}

This paper was supported by Wonkwang University, Iksan, Korea in 2018.

\section{REFERENCES}

1. Buchanan MA, Wilkinson JM, Fitzgerald JE, Prinsley PR. Is golf bad for your hearing? BMJ. 2008 Dec;337:a2835.

2. Zhao F, Bardsley B. Real-ear acoustical characteristics of impulse sound generated by golf drivers and the estimated risk to hearing: a cross-sectional study. BMJ Open. 2014 Jan;4(1):e003517.

3. Kim YH, Kim YC, Lee JH, An YH, Park KT, Kang KM, et al. Analysis of impact noise induced by hitting of titanium head golf driver. Eur Arch Otorhinolaryngol. 2014 Nov;271(11):2885-90.

4. Adelman C, Weinberger JM, Kriksunov L, Sohmer H. Effects of furosemide on the hearing loss induced by impulse noise. J Occup Med Toxicol. 2011 May;6(1):14.

5. Bielefeld EC. Reduction in impulse noise-induced permanent threshold shift with intracochlear application of an NADPH oxidase inhibitor. J Am Acad Audiol. 2013 Jun;24(6):461-73.

6. Kopke RD, Jackson RL, Coleman JK, Liu J, Bielefeld EC, Balough BJ. NAC for noise: from the bench top to the clinic. Hear Res. 2007 Apr;226(1-2):114-25.

7. Le Prell CG, Hughes LF, Miller JM. Free radical scavengers vitamins A, C, and E plus magnesium reduce noise trauma. Free Radic Biol Med. 2007 May;42(9):1454-63.

8. Yamashita D, Jiang HY, Le Prell CG, Schacht J, Miller JM. Post-exposure treatment attenuates noise-induced hearing loss. Neuroscience. 2005;134(2):633-42.

9. Henderson D, Bielefeld EC, Harris KC, Hu BH. The role of oxidative stress in noise-induced hearing loss. Ear Hear. 2006 Feb;27(1):1-19.

10. Maeda Y, Fukushima K, Omichi R, Kariya S, Nishizaki K.Time courses of changes in phospho- and total- MAP kinases in the cochlea after intense noise exposure. PLoS One. 2013;8(3):e58775.

11. Hodge DC, Price GR. Hearing damage risk criteria. In: Lipscomb $\mathrm{DM}$, editor. Noise and audiology (perspectives in audiology series). Baltimore (ME): University Park Press; 1978. p. 167-91.

12. Clifford RE, Rogers RA. Impulse noise: theoretical solutions to the quandary of cochlear protection. Ann Otol Rhinol Laryngol. 2009 Jun;118(6):417-27.

13. Starck J,Toppila E, Pyykko I. Impulse noise and risk criteria. Noise Health. 2003 Jul-Sep;5(20):63-73.

14. Akhmetzianov IM, Zinkin VI, Logatkin SM, Petreev IV, Kuznetsov SM, Dragan SP. Impulse noise at shooting from small arms and close combat weapon as the factor of military work. Voen Med Zh. 2012 Jun;333(6):52-8.

15. Rezaee M, Mojtahed M, Ghasemi M, Saedi B. Assessment of impulse noise level and acoustic trauma in military personnel. Trauma Mon. 2012 Jan;16(4):182-7.

16. Lwow F, Jozkow P, Medras M. Occupational exposure to impulse noise associated with shooting. Int J Occup Saf Ergon. 2011;17(1): 69-77.

17. Forget P. Assessment of mean auditory hazard incurred by occupational exposure to impulse noise. Eur Ann Otorhinolaryngol Head Neck Dis. 2011 Jan;128(1):14-7.

18. Sendowski I,Abaamrane L, Raffin F, Cros A, Clarencon D. Therapeutic efficacy of intra-cochlear administration of methylprednisolone after acoustic trauma caused by gunshot noise in guinea pigs. Hear Res. 2006 Nov;221(1-2):119-27.

19. Flamme GA, Liebe K, Wong A. Estimates of the auditory risk from outdoor impulse noise. I: Firecrackers. Noise Health. 2009 Oct-Dec; 11(45):223-30.

20. Flamme GA, Wong A, Liebe K, Lynd J. Estimates of auditory risk from outdoor impulse noise. II: Civilian firearms. Noise Health. 2009 Oct-Dec;11(45):231-42.

21. Wu CC, Young YH. Ten-year longitudinal study of the effect of impulse noise exposure from gunshot on inner ear function. Int J Audiol. 2009 Sep;48(9):655-60.

22. Abaamrane L, Raffin F, Gal M, Avan P, Sendowski I. Long-term administration of magnesium after acoustic trauma caused by gunshot noise in guinea pigs. Hear Res. 2009 Jan;247(2):137-45.

23. Levine S, Hofstetter P, Zheng XY, Henderson D. Duration and peak level as co-factors in hearing loss from exposure to impact noise. Scand Audiol Suppl. 1998;48:27-36.

24. Danielson R, Henderson D, Gratton MA, Bianchi L, Salvi R. The importance of "temporal pattern" in traumatic impulse noise exposures. J Acoust Soc Am. 1991 Jul;90(1):209-18.

25. Pelausa EO, Abel SM, Simard J, Dempsey I. Prevention of noise-induced hearing loss in the Canadian military. J Otolaryngol. 1995 Oct;24(5):271-80.

26. Suvorov GA, Denisov EI,Antipin VG, Kharitonov VI, Starck Iu, Pyykko I, et al. Effects of peak levels and number of noise impulses on hearing among forge hammering workers. Med Tr Prom Ekol. 2002; (12):12-6.

27. Neitzel R, Seixas N. The effectiveness of hearing protection among construction workers. J Occup Environ Hyg. 2005 Apr;2(4):227-38.

28. Pourbakht A, Yamasoba T. Cochlear damage caused by continuous and intermittent noise exposure. Hear Res. 2003 Apr;178(1-2):70-8.

29. Nilsson P, Rydmarker S, Grenner J. Impulse noise and continuous noise of equivalent frequency spectrum and total sound energy. Acta Otolaryngol Suppl. 1987;441:45-58.

30. Kil J, Pierce C, Tran H, Gu R, Lynch ED. Ebselen treatment reduces noise induced hearing loss via the mimicry and induction of glutathione peroxidase. Hear Res. 2007 Apr;226(1-2):44-51.

31. Lynch ED, Gu R, Pierce C, Kil J. Ebselen-mediated protection from single and repeated noise exposure in rat. Laryngoscope. 2004 Feb; 114(2):333-7.

32. Fechter LD, Klis SF, Shirwany NA, Moore TG, Rao DB. Acrylonitrile produces transient cochlear function loss and potentiates permanent noise-induced hearing loss. Toxicol Sci. 2003 Sep;75(1):117-23.

33. Rabinowitz PM, Pierce Wise J Sr, Hur Mobo B, Antonucci PG, Powell C, Slade M. Antioxidant status and hearing function in noise-exposed workers. Hear Res. 2002 Nov;173(1-2):164-71.

34. Yamashita D, Jiang HY, Schacht J, Miller JM. Delayed production of free radicals following noise exposure. Brain Res. 2004 Sep;1019(12):201-9.

35. Elsayed NM. Toxicology of blast overpressure. Toxicology. 1997 Jul; 121(1):1-15.

36. Huie RE, Padmaja S. The reaction of no with superoxide. Free Radic Res Commun. 1993;18(4):195-9.

37. Linss V, Emmerich E, Richter F, Linss W. Is there a close relationship between changes in amplitudes of distortion product otoacoustic emissions and hair cell damage after exposure to realistic industrial noise in guinea pigs? Eur Arch Otorhinolaryngol. 2005 Jun;262(6): 488-95.

38. Roberto M, Hamernik RP, Turrentine GA. Damage of the auditory system associated with acute blast trauma. Ann Otol Rhinol Laryngol Suppl. 1989 May;140:23-34.

39. Henderson D, McFadden SL, Liu CC, Hight N, Zheng XY. The role of antioxidants in protection from impulse noise. Ann NY Acad Sci. 1999 Nov;884(1):368-80.

40. Chi FL, Yang MQ, Zhou YD, Wang B. Therapeutic efficacy of topical application of dexamethasone to the round window niche after acoustic trauma caused by intensive impulse noise in guinea pigs. J Laryn- 
gol Otol. 2011 Jul;125(7):673-85

41. Chang MY, Rhee J, Kim SH, Kim YH. The protective effect of Egb 761 against 3-nitropropionic acid-induced hearing loss: the role of sirtuin 1. Clin Exp Otorhinolaryngol. 2018 Mar;11(1):9-16.

42. Tian C, Kim YH, Kim YC, Park KT, Kim SW, Kim YJ, et al. Korean red ginseng ameliorates acute 3-nitropropionic acid-induced cochle- ar damage in mice. Neurotoxicology. 2013 Jan;34:42-50.

43. Kim YH, Song JJ, Kim YC, Park KT, Lee JH, Choi JM, et al. Geranylgeranylacetone ameliorates acute cochlear damage caused by 3-nitropropionic acid. Neurotoxicology. 2010 Jun;31(3):317-25.

44. Ohinata Y, YamasobaT, Schacht J, Miller JM. Glutathione limits noiseinduced hearing loss. Hear Res. 2000 Aug;146(1-2):28-34. 